\title{
Paget Disease of the Breast with Invasive Ductal Carcinoma
}

National Cancer Institute

\section{Source}

National Cancer Institute. Paget Disease of the Breast with Invasive Ductal Carcinoma. NCl Thesaurus. Code C7951.

Paget disease involving the skin overlying the mammary gland, accompanied by invasive ductal breast carcinoma. 